\title{
Papers
}

\section{Oral pristinamycin versus standard penicillin regimen to treat erysipelas in adults: randomised, non-inferiority, open trial}

\author{
Philippe Bernard, Olivier Chosidow, Loïc Vaillant on behalf of the French Erysipelas Study Group
}

\begin{abstract}
Objective To assess the efficacy and safety of oral pristinamycin versus intravenous then oral penicillin to treat erysipelas in patients in hospital.

Design Multicentre, parallel group, open labelled, randomised non-inferiority trial.

Setting 22 French hospitals.

Participants 289 adults admitted to hospital with erysipelas.

Results At follow up (day 25-45) the cure rate (primary efficacy end point) for the per protocol populations was $81 \%(83 / 102)$ for pristinamycin and $67 \%(68 / 102)$ for penicillin. The planned interim analysis (global one sided type I error $5 \%$ ) showed that the one sided $97.06 \%$ confidence interval of the observed difference (pristinamycin - penicillin) between cure rates $(3.3 \%$ to $\infty$ ) exceeded the $-10 \%$ non-inferiority threshold. For the intention to treat populations the cure rate at follow up was $65 \%$ (90/138) for pristinamycin and 53\% (79/150) for penicillin, with the one sided $97.06 \%$ confidence interval of the observed difference between cure rates $(1.7 \%$ to $\infty)$ exceeding the $-10 \%$ non-inferiority threshold. That the lower limit of the confidence interval exceeded the $-10 \%$ threshold and was also $>0$ supports the hypothesis that pristinamycin is significantly superior at the $5 \%$ level. More adverse events related to treatment, as assessed by the investigators, were reported in the pristinamycin group than in the penicillin group. Most adverse events involved the gastrointestinal tract (nausea, vomiting, and diarrhoea) but were minor and usually did not require discontinuation of treatment.

Conclusion Pristinamycin could be an alternative to the standard intravenous then oral penicillin regimen used to treat erysipelas in adults in hospital, with the advantages of oral first line therapy.
\end{abstract}

\section{Introduction}

Erysipelas is an acute superficial dermal-hypodermal infection (cellulitis) that usually affects the leg and is commonly caused by streptococci. ${ }^{1-6}$ Our prospective case-control study highlighted the major role of local risk factors, principally lymphoedema and site of entry. ${ }^{6}$ Erysipelas is severe but not usually life threaten- ing. Although 10-20\% of patients admitted to hospital may develop early local complications (abscess, superficial or deep gangrene), systemic complications, including septicaemia, are rare. ${ }^{3-10}$ Erysipelas can also be treated on an outpatient basis. ${ }^{11}$

Although regimens vary, the standard treatment is intravenous penicillin G. ${ }^{35781012-14}$ Unfortunately, intravenous treatment is painful and time consuming, and side effects, including local complications, are not uncommon. ${ }^{379}$ Furthermore, it requires admission to hospital. To date, only a few studies have evaluated the efficacy of oral penicillin ${ }^{12}$ or macrolides ${ }^{14}$ versus standard penicillin to treat erysipelas or acute cellulitis. Pristinamycin, a natural streptogramin commonly used in some European countries, particularly France, is especially active against Streptococcus pyogenes (minimum inhibitory concentration $\leqslant 0.12 \mathrm{mg} / \mathrm{l}) .{ }^{15} \mathrm{In}$ a preliminary open study, pristinamycin cured $86 \%$ of adult patients admitted to hospital with erysipelas. ${ }^{16}$

We undertook a large scale investigation to compare the efficacies of oral pristinamycin and intravenous then oral penicillin in treating hospital patients with erysipelas.

\section{Patients and methods}

\section{Study design}

The study was a randomised open label, parallel group clinical trial designed to assess the non-inferiority of oral pristinamycin versus a standard intravenous then oral penicillin regimen. It took place from August 1998 to November 2000 in 22 dermatology centres in France. It was approved by an institutional ethics committee and performed in accordance with good clinical practice guidelines.

We used a non-inferiority approach to investigate whether pristinamycin is not less effective than the reference treatment. Therefore, the one sided confidence interval of the difference in the cure rates between pristinamycin and penicillin had to be entirely above the prespecified non-inferiority threshold $(-10 \%){ }^{17}$

Patients were randomly assigned to 14 days' treatment with either oral pristinamycin $(1 \mathrm{~g}$ three times a day) or benzylpenicillin (18 MU/day in six infusions) until their temperature was normal and then oral phenoxymethylpenicillin (2 MU three times a day). The randomisation was centralised and balanced
Department of
Dermatology,
Hôpital
Robert-Debré,
51092 Reims,
France
Philippe Bernard
professor
Department of
Internal Medicine,
Hôpital
Pitié-Salpêtrière,
75013 Paris, France
Olivier Chosidow
professor
Department of
Dermatology,
Hôpital Trousseau,
37044 Tours,
France
Loïc Vaillant
professor
Correspondence to:
P Bernard
pbernard@
chu-reims.fr
bmj.com 2002;325:864 
by centre. The allocation sequence was generated with a computer list of random set numbers, stratified by centre and blocked. Containers numbered in increasing values were used to implement the random allocation sequence. All study medications were supplied by Laboratoire Aventis.

\section{Patients}

All adults with erysipelas who were admitted to hospital in each of the participating centre were eligible in the study. Erysipelas was defined clinically as acute well delineated dermal-hypodermal inflammation that had lasted less than five days, was associated with fever $\left(\geqslant 38.5^{\circ} \mathrm{C}\right)$ or chills, ${ }^{6}$ and corresponded to superficial non-necrotising cellulitis as defined elsewhere. ${ }^{4-6} \mathrm{We}$ excluded patients in whom necrotising cellulitis or fasciitis, or a subcutaneous abscess was suspected; a bite or scratch had occurred during the preceding seven days; or HIV infection was known. We also excluded any patients who had previously received benzylpenicillin or pristinamycin treatment for over 12 hours; had known allergy to synergistins or $\beta$ lactams; had renal or hepatic insufficiency; or were taking steroids or immunosuppressant drugs. The use of antipyretics $>500$ $\mathrm{mg} /$ day or any other antibiotic was prohibited during the study.

To assess the local severity of the infection we calculated a clinical score describing the oedema, erythema, and pain of cutaneous plaque using a three point scale $(0=$ absent, $1=$ moderate, $2=$ severe). To be included, each patient had to have a total clinical score $\geqslant 3$ and to provide a written informed consent.

\section{Baseline and follow up assessments}

We carried out assessments at enrolment, 48-72 hours after starting treatment, during treatment (days 4-10), at the end of treatment (day 14-17), and at follow up (day 25-45). At each visit participants underwent a complete physical examination, and we calculated a severity score. Patients whose local or general status had deteriorated could be withdrawn from the trial and the treatment was considered to have failed.

At enrolment we tested blood sample and swabs from probable entry sites for aerobic and anaerobic bacteria. Laboratory tests included complete blood count, $\mathrm{C}$ reactive protein, and blood chemistries. We used results of antistreptolysin $\mathrm{O}$ and antistreptodornase tests as supportive data.

\section{Primary and secondary end points}

All randomised patients were included in the intention to treat analysis and safety analysis populations. Patients without any major protocol violation were included in the per protocol population.

The primary end point was the clinical cure rate determined at follow up for the per protocol population. We defined clinical cure as body temperature $<37.5^{\circ} \mathrm{C}$, complete regression of local or general signs of severity, and disappearance of the cutaneous plaque (final clinical score $=0$ ). We defined clinical failure as the presence of at least one of the following: body temperature $>37.9^{\circ} \mathrm{C}$, persistent or recurrent cutaneous plaque, local or general signs of severity requiring new treatment, or intake of another systemic antibiotic between end of treatment and follow up for more than two calendar days. All other cases had to be reviewed blindly by the scientific committee $(\mathrm{PB}, \mathrm{OC}$, LV).

The secondary efficacy variables were the clinical cure rate determined at follow up for the intention to treat population and the clinical success rate determined at the end of treatment for the intention to treat and per protocol populations.

\section{Statistical analyses}

Our main objective was to assess the non-inferiority of pristinamycin versus penicillin, and we based our calculation of sample size on the primary end point. We assumed an $85 \%$ cure rate at follow up for both groups and a non-inferiority margin of $10 \%$.

To maintain a global one sided $\alpha$ of $5 \%$ we set the error risk at $\alpha 1=2.94 \%$ for the interim analysis and $\alpha 2=2.94 \%$ for the final analysis, according to Pocock's adjustment. ${ }^{18}$ On the basis of these hypotheses and a conservative calculation based solely on the final analysis (taking into account a nominal type I error of $2.94 \%$ ) we considered that $2 \times 190$ assessable patients would suffice to confirm the non-inferiority of pristinamycin with a power of $80 \%$. We assumed an estimated ratio of $10 \%$ non-assessable patients and therefore defined the sample size as 420 patients. We planned to carry out an interim analysis once we had enroled about $2 \times 95$ assessable patients.

We compared primary and secondary efficacy variables between the two treatment groups using a one sided non-inferiority test: the lower limit of the confidence interval of the difference in cure rates between pristinamycin and penicillin had to exceed the predefined threshold of $-10 \% .{ }^{17}$ The non-inferiority margin for all criteria was set at $10 \%$. We calculated estimates and standard errors of the effects of

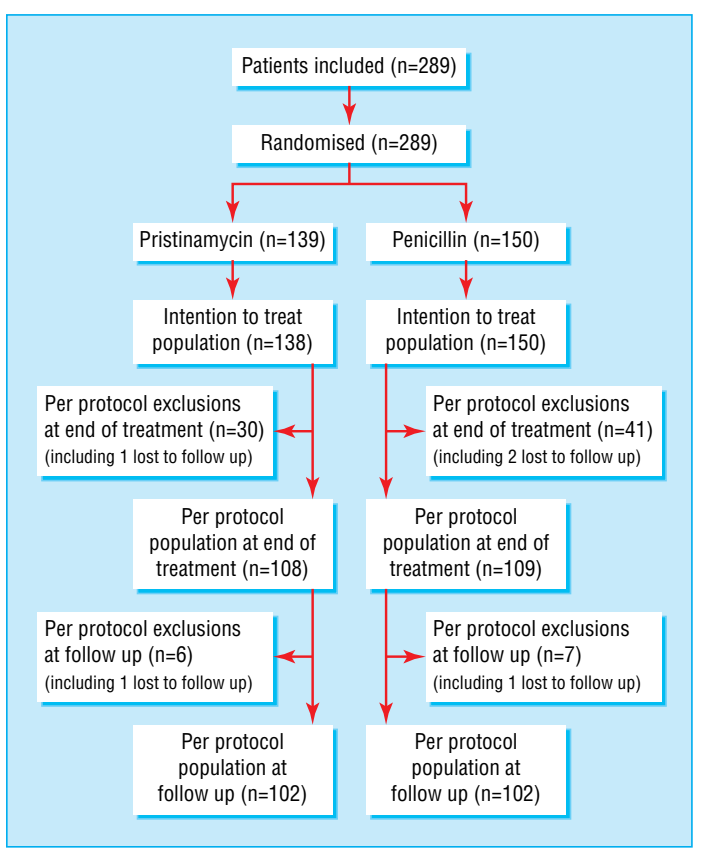

Trial regimen ( ${ }^{*}$ one patient randomised twice in error but was included only once in efficacy analysis). Exclusions from per protocol at end of treatment were because of non-compliance with treatment, prohibited treatment used during study, discontinuation of treatment due to adverse event, or missing data (patient may have had one or more major protocol violation). Exclusions at follow up were because of missing data or prohibited treatment used during study (patient may have had one or more major protocol violation) 
Table 1 Baseline characteristics of patients (intention to treat populations). Figures are numbers (percentage) of patients unless stated otherwise

\begin{tabular}{lcc} 
Characteristic & $\begin{array}{c}\text { Pristinamycin } \\
(\mathbf{n = 1 3 8 )}\end{array}$ & Penicillin (n=150) \\
\hline No of men & 78 & 74 \\
\hline Mean (SD) age (years) & $57(18)$ & $60(18)$ \\
\hline Mean (SD) weight (kg) & $81(20)$ & $80(20)$ \\
\hline Associated disease: & & \\
\hline At least one concurrent disease & $83(60)$ & $88(59)$ \\
\hline Venous insufficiency & $59(43)$ & $69(46)$ \\
\hline Cardiac failure & $18(13)$ & $9(6)$ \\
\hline Type 1 diabetes & $2(1)$ & $2(1)$ \\
\hline Type 2 diabetes & $19(14)$ & $23(15)$ \\
\hline Alcoholism & $17(12)$ & $8(5)$ \\
\hline Location of erysipelas: & & $145(97)$ \\
\hline Lower limb & $126(91)$ & $5(3)$ \\
\hline Other location & $12(9)$ & $141(94)$ \\
\hline Probable portal of entry: & $131(95)$ & $94(67)$ \\
\hline Toe web intertrigo & $78(60)$ & $36(26)$ \\
\hline Wound & $41(31)$ & $26(18)$ \\
\hline Leg ulcer & $16(12)$ & $53(37)$ \\
\hline Other lesion & $44(33)$ & $65(43)$ \\
\hline Severity of erysipelas: & & $102(68)$ \\
\hline Severe erythema & $81(59)$ & $60(40)$ \\
\hline Severe pain & $54(39)$ & $51(37)$ \\
\hline Severe oedema & $4.3(1.1)$ & \\
\hline Mean (SD) total clinical score & & \\
\hline
\end{tabular}

pristinamycin versus penicillin. For safety tests we used Fisher's exact tests, and we considered two sided $\mathrm{P}$ values $<5 \%$ as significant.

\section{Results}

\section{Patients}

We performed the interim analysis when we had included 289 patients. At follow up we were able to evaluate 204 patients (102 in each arm); randomisation had assigned 139 patients to receive pristinamycin and 150 to receive penicillin (figure). With the exception of one patient wrongly included twice, all randomised patients were included in the intention to treat population and received at least one dose of the study medication.

For the 288 patients comprising the intention to treat population (mean age 59 (SD 18) years; range 18-96 years) the baseline characteristics studied were similar according to the assigned antibiotic (table 1). Microbiological data were available for 257/288 (89\%) patients. Streptococcus species were isolated from probable entry sites for 64/257 (25\%) patients and from blood cultures for $5 / 257$ (2\%).

\section{Efficacy}

\section{Treatment}

The per protocol populations at follow up (figure) included 102/138 (74\%) patients in the pristinamycin group and 102/150 (68\%) in the penicillin group, with, respectively, 108/138 (78\%) and 109/150 (73\%) retained in the end of treatment per protocol populations.

For the pristinamycin and penicillin groups, respectively, the mean (SD) duration of treatment was 15.0 (SD 1.0) and 14.8 (SD 1.4) days (intention to treat populations) and the mean duration of hospital stay was 11.0 (SD 8.2) and 11.4 (SD 8.1) days. Mean clinical severity scores evolved similarly for both groups (table 2). Clinical signs suggestive of necrotising cellulitis occurred in $9 / 138(7 \%)$ patients in the pristinamycin group and $14 / 150(9 \%)$ in the penicillin group $(\mathrm{P}=0.38)$.

\section{Primary efficacy variable}

At follow up the respective cure rates for the pristinamycin and penicillin per protocol populations were $81 \%(83 / 102)$ and $67 \%(68 / 102)$ (table 3$)$, with the lower limit of the one sided $97.06 \%$ confidence interval for the observed difference between them $(3.3 \%$ to $\infty)$ exceeding the $-10 \%$ non-inferiority threshold.

For the per protocol population, the pristinamycin effect was estimated to be +14.7 (SE 6.1\%) (estimation of the difference).

\section{Secondary efficacy variables}

For the pristinamycin and penicillin intention to treat populations, the respective cure rate at follow up was $65 \%(90 / 138)$ and 53\% (79/150), with the lower limit of the one sided $97.06 \%$ confidence interval for the observed difference between them $(1.7 \%$ to $\infty)$ exceeding the $-10 \%$ non-inferiority threshold (table 3 ).

For the intention to treat population, the pristinamycin effect was estimated (as above) to be +12.6 ( $\mathrm{SE}$ $5.9 \%)$.

Because this interim analysis established the non-inferiority of pristinamycin compared with penicillin, it became the final one. Moreover, the $14.7 \%$ difference in favour of pristinamycin over penicillin on the principal assessment criterion and the confidence interval support the hypothesis of a superiority of pristinamycin.

The clinical success rates at the end of treatment for per protocol and intention to treat populations also showed the non-inferiority of pristinamycin because the lower limit of the one sided $97.06 \%$ confidence interval of the observed difference between them exceeded the predefined non-inferiority threshold of $-10 \%$ (table 3).

\section{Safety}

Table 4 summarises any adverse events reported by investigators. Adverse events related to treatment were more common in the pristinamycin group $(\mathrm{P}=0.034)$. They were mostly mild or moderate and mainly involved the gastrointestinal tract (table 4). The proportions of adverse events necessitating discontinuation of the study medication were similar for the two groups $(\mathrm{P}=0.174)$.

Two patients in the penicillin group experienced a severe adverse event-that is, leucopenia or erythema multiforme- that were considered possibly related to the study medication.

Table 2 Evolution of mean total clinical score at five visits (intention to treat populations)

\begin{tabular}{lccccc} 
Antibiotic & Enrolment & $\mathbf{4 8 - 7 2}$ hours & $\mathbf{4 - 1 0}$ days & End of treatment & Follow up \\
\hline $\begin{array}{l}\text { Pristinamycin } \\
\text { No of patients }\end{array}$ & 138 & 131 & 126 & 120 & 122 \\
\hline Mean (SD) score & $4.3(1.1)$ & $2.4(1.3)$ & $1.3(1.1)$ & $0.9(1.1)$ & $0.4(0.9)$ \\
\hline Penicillin & & & & \\
\hline No of patients & 150 & 144 & 123 & 115 & 120 \\
\hline Mean (SD) score & $4.5(1.0)$ & $2.8(1.4)$ & $1.6(1.4)$ & $0.9(1.2)$ & $0.5(1.0)$ \\
\hline
\end{tabular}


Table 3 Clinical responses at follow up (day 25-45) and at end of treatment (day 14-17)

\begin{tabular}{lllll} 
Response & Pristinamycin & Penicillin & $\mathbf{9 7 . 0 6 \%} \mathbf{C l}$ (one sided) & $\mathbf{9 4 . 1 2 \%}$ Cl (two sided) \\
\hline Cure rate at follow up : & & & & \\
\hline Per protocol population & $81 \%(83 / 102)$ & $67 \%(68 / 102)$ & $(3.3 \%$ to $\infty)$ & $(3.3 \%$ to $26.1 \%)$ \\
\hline Intention to treat population & $65 \%(90 / 138)$ & $53 \%(79 / 150)$ & $(1.7 \%$ to $\infty)$ & $(1.7 \%$ to $23.4 \%)$ \\
\hline Clinical success rate at end of treatment†: & & & & \\
\hline Per protocol population & $89 \%(96 / 108)$ & $74 \%(81 / 109)$ & $(4.8 \%$ to $\infty)$ & $(4.8 \%$ to $24.3 \%)$ \\
\hline Intention to treat population & $74 \%(102 / 138)$ & $63 \%(95 / 150)$ & $(0.3 \%$ to $\infty)$ & $(0.3 \%$ to $20.8 \%)$ \\
\hline
\end{tabular}

*Primary end point.

tCure and lesion regression.

\section{Discussion}

Previous studies of efficacy

This study is the first large scale trial to show clearly that oral treatment can replace intravenous then oral penicillin to treat erysipelas in patients in hospital. The per protocol populations achieved cure rates at follow up of $81 \%$ with pristinamycin and $67 \%$ with penicillin. At present, the reference treatment for erysipelas is penicillin, usually administered intravenously for at least 10 days. ${ }^{357810121419}$ Surprisingly, randomised prospective trials to assess the efficacy of intravenous penicillin against erysipelas are rare in the literature. ${ }^{12}{ }^{14} 19$ Other than those trials, penicillin regimens for erysipelas have been derived from retrospective studies. ${ }^{37810}$ The French consensus conference recommendation to prescribe oral amoxicillin for erysipelas was based on clinical practice but not supported by adequate clinical trials. ${ }^{20}$ The first comparative study assessing the efficacy of oral antibiotics (phenoxymethylpenicillin, alone or combined with flucloxacillin) against erysipelas included 73 patients. ${ }^{12}$ However, in that study overall efficacy rates were not specified and 10 patients given clindamycin were not excluded from the analysis. A multicentre open label randomised study that compared oral roxithromycin with penicillin obtained overall efficacy rates of $84 \%$ and $76 \%$, respectively, but too few patients had been included $(n=69)$ to assess their relative efficacies. ${ }^{14}$

Design and results of present trial

Although we would have preferred to use a double blind, double placebo design, this study is the first in erysipelas or cellulitis in which the design corresponds

Table 4 Adverse events observed according to assigned antibiotic (intention to treat populations). Figures are numbers (percentage) of patients

\begin{tabular}{lcc} 
& $\begin{array}{c}\text { Pristinamycin } \\
(\mathbf{n = 1 3 9 )}\end{array}$ & $\begin{array}{c}\text { Penicillin } \\
(\mathbf{n}=\mathbf{1 5 0})\end{array}$ \\
\hline Patients with $\geqslant 1$ event & $75(54)$ & $83(55)$ \\
\hline Patients with at least one possible event related to drug* & $39(28)$ & $26(17)$ \\
\hline Patients withdrawn prematurely & $15(11)$ & $25(\mathbf{1 7 )}$ \\
\hline Event related to drug related in $\geqslant 2$ patients: & 2 & 2 \\
\hline Abdominal pain & 2 & 0 \\
\hline Gastrointestinal disorder & 3 & 0 \\
\hline Dyspepsia & 4 & 0 \\
\hline Nausea & 7 & 0 \\
\hline Vomiting & 14 & 4 \\
\hline Diarrhoea & 4 & 6 \\
\hline Liver function test abnormality & 0 & 2 \\
\hline Allergic reaction & 4 & 4 \\
\hline Rash & 2 & 0 \\
\hline Sweats & 1 & 1 \\
\hline Moniliasis & 1 & 1 \\
\hline Urticaria & & \\
\hline
\end{tabular}

*As stated by investigator. to that of a non-inferiority trial, simultaneously evaluating a large number of patients and using strict inclusion criteria and statistical analysis based on the confidence interval. As recommended for noninferiority trials, the analysis was conducted on both per protocol and intention to treat populations. ${ }^{17}{ }^{21}$ As the study design was more explanatory than pragmatic and our main aim was to assess the non-inferiority of pristinamycin versus penicillin, the primary end point relies on the per protocol analysis. ${ }^{17}$ In addition, full application of the intention to treat analysis will be possible only once complete outcome data become available for all randomised patients. ${ }^{22}$

Most of our non-assessable patients were excluded from the per protocol analysis because of adverse events leading to premature withdrawal (see below), missing data, or use of prohibited treatment. Indeed, our intention to treat analysis also confirmed the noninferiority of pristinamycin as inclusion of noncompliers decreased the cure rates to similar extents in both groups. Moreover, the $14.7 \%$ difference in favour of pristinamycin over penicillin for the principal assessment criterion and the related two sided $97.06 \%$ confidence interval (intention to treat: $0.03 \%$ to $25.1 \%$; per protocol: $1.5 \%$ to $27.9 \%$ ) support the hypothesis of a significant difference in favour of pristinamycin. It is therefore possible to confirm a significant difference at the $5 \%$ level in accordance with the guidelines of the Committee for Proprietary Medicinal Products. ${ }^{17}$

\section{Future directions of treatment}

The numbers of withdrawals due to adverse events were similar for both regimens. As expected, more minor gastrointestinal symptoms without clinical consequence occurred in the pristinamycin group. Because our data show that treatment of erysipelas with oral pristinamycin is not clinically inferior to intravenous then oral penicillin some practical and economic points merit consideration. Currently, pristinamycin is marketed only in some European countries. It is commonly used in France to treat erysipelas ${ }^{11}$ and superficial pyodermas ${ }^{23}$ in outpatients. In addition, because our strict inclusion criteria excluded patients with minor or severe erysipelas (that is, with systemic or local signs of severity) the patients enrolled represent only a subclass of those admitted to hospital. These results indicate that patients with uncomplicated erysipelas can be directly treated orally as outpatients, thereby preventing nosocomial complications. ${ }^{24}$ Our results show that pristinamycin could be an alternative to intravenous then oral penicillin to treat erysipelas in adult inpatients, with the advantage of oral first line treatment. Whether this therapeutic strategy is valid for outpatients, both from clinical and pharmacoeconomic points of view, needs to be investigated. 


\section{What is already known on this topic}

The reference treatment for erysipelas is intravenous penicillin, which requires admission to hospital

Few studies have evaluated the efficacy of oral treatment for erysipelas

\section{What this study adds}

Oral pristinamycin is at least as effective as intravenous then oral penicillin to treat erysipelas in adult inpatients, with the advantage of oral first line treatment

We are grateful to the members of the French Erysipelas Study Group: K Bernardeau, O Estines (Reims); H Dega (Paris); L Martin (Tours); S Aractingi, V Vaisse (Paris); J-M Bonnetblanc, C Bedane, M-L Bouyssou-Gauthier (Limoges); F Cambazard, J-L Perrot, L Misery, J-L Michel (Saint-Etienne); F Carsuzaa, B Guennoc (Toulon); P Combemale, M Dupin, J-L Estival (Lyons); E Grosshans, C Chartier (Strasbourg); J-C Guillaume, F Grange, D Couilliet (Colmar); B Guillot, M Dandurand (Nimes); P Humbert, B Faivre, Bizouard (Besançon); P Joly, P Young, N Cordel (Rouen); B Labeille, C Moulin (Valence); D Lambert, J-P Touraud, A Claude (Dijon); R Laurent, C Drobacheff, D Salard (Besançon); J Revuz, A Bouloc, L Le Cleach (Créteil); B Sassolas, J Lonceint (Brest); J-L Schmutz, F Granel (Nancy); M Sigal-Grindberg, A Petit, C Iborra (Argenteuil); P Souteyrand, S Parent, M D'Incan (Clermont-Ferrand); and P Thomas, E Delaporte, A-C Cottentin, M Segard (Lille).

We are also grateful to C Jean, Y Boutalbi, and O Lescale (Laboratoire Aventis) for their help in the data analysis and preparation of the manuscript and P Joffre-Malamas (Laboratoire Aventis) for the statistical analysis.

Contributors: All the authors participated in the protocol design, analysis, interpretation of the data, and writing the paper. Funding: Aventis (Paris, France).

Competing interests: $\mathrm{PB}, \mathrm{OC}$, and $\mathrm{LV}$ have received fees from Aventis for helping to design and organise the study and review difficult cases (steering committee).

1 Bernard P, Bedane C, Mounier M, Denis F, Catanzano G, Bonnetblanc JM. Streptococcal cause of erysipelas and cellulitis in adults: a microbiologic study. Arch Dermatol 1989;125:779-82.

2 Duvanel T, Auckenthaler R, Rohner P, Harms M, Saurat JH. Quantitative cultures of biopsy specimens from cutaneous cellulitis. Arch Intern Med 1989;149:293-6.
3 Chartier C, Grosshans E. Erysipelas. Int J Dermatol 1990;29:459-67.

4 Swartz MN. Cellulitis and subcutaneous tissue infections. In: Mandell GL, Bennett JE, Dolin R, eds.Principles and practice of infectious diseases. New York: Churchill Livingstone 1995:909-15.

5 Bisno AL, Stevens DL. Streptococcal infections of the skin and soft tissues. N Engl J Med 1996;334:240-5.

6 Dupuy A, Benchikhi H, Roujeau JC, Bernard P, Vaillant L, Chosidow O, et al. Risk factors for erysipelas of the leg (cellulitis): case-control study. BMJ 1999;318:1591-4

7 Jorup-Rönström C. Epidemiological, bacteriological and complicating features of erysipelas. Scand J Infect Dis 1986;18:519-24.

8 Chartier C, Grosshans E. Erysipelas: an update. Int J Dermatol 1996;35:779-81.

9 Eriksson B, Jorup-Rönström C, Karkkonen K, Sjöblom AC, Blom SE Erysipelas: clinical and bacteriologic spectrum and serological aspects. Clin Infect Dis 1996;23:1091-8.

10 Ronnen P, Suster S, Schewach-Millet M, Modan M. Erysipelas: changing faces. Int J Dermatol 1985;24:169-72.

11 Kopp M, Schmit JL, Bernard P. Prise en charge des dermohypodermites bactériennes par les médecins généralistes: enquête de pratique et étude prospective. Ann Dermatol Vénéréol 2001;128:338-44.

12 Jorup-Rönström C, Britton S, Gavlevick A, Gunnarson AC. The course, costs and complications of oral versus intravenous penicillin therapy of erysipelas. Infection 1984;12:390-4.

13 Schmit JL. Enquête prospective. Erysipèle et cellulites nécrosantes: quelle prise en charge en milieu hospitalier? Ann Dermatol Vénéréol 2001;128:334-7.

14 Bernard P, Plantin P, Roger H, Sassolas B, Villaret E, Legrain V, et al Roxithromycin versus penicillin in the treatment of erysipelas in adults: a comparative study. Br J Dermatol 1992;127:155-9.

15 Reverdy ME, Coignard S, Dubreuil L, Bouvet A, Fleurette J. Activité in vitro de la cloxacilline sur les staphylocoques, les streptocoques du groupe A et Clostridium perfringens. Index inhibiteurs sériques. Méd Mal Infect 1994;24:898-905.

16 Bernard P, Risse L, Bonnetblanc JM. Traitement des dermohypodermites aiguës bactériennes de l'adulte par la pristinamycine: étude ouverte chez 42 patients. Ann Dermatol Vénéréol 1996;123:16-20.

17 Committee for Proprietary Medicinal Products, European Agency for the Evaluation of Medicinal Products. Points to consider on switching between superiority and non-inferiority. London: EAEMP, 2000 (CPMP/EWP/482 99.)

18 Pocock SJ. Group sequential methods in the design and analysis of clinical trials. Biometrika 1977;64:191-9.

19 Bergkvist PI, Sjöbeck K. Antibiotic and prednisolone therapy of erysipelas: a randomised, double-blind, placebo-controlled study. Scand J Infect Dis 1997;29:377-8,

20 Erysipèle et fasciite nécrosante: prise en charge. Conférence de consensus. Ann Dermatol Vénéréol 2000;127:1118-37.

21 Siegel JP. Equivalence and noninferiority trials. Am Heart $J$ 2000;139(suppl):S167-70.

22 Jones B, Jarvis P, Lewis JA, Ebbutt AF. Trials to assess equivalence in medical research: are they supported by the evidence? $B M$ J 1996;313:36-9.

23 Bernard P, Vaillant L, Martin C, Beylot C, Quentin R, Touron D. Pristinamycine (Pyostacine 500) versus oxacilline dans le traitement des pyodermites superficielles: résultats d'une étude randomisée multicentrique chez 293 malades ambulatoires. Ann Dermatol Vénéréol trique chez $1997 ; 124: 384-9$

24 Jorup-Rönström C, Britton S. The nosocomial component of medical care. Scand J Infect Dis 1982;36 (suppl):S150-6.

(Accepted 6June 2002) 